\title{
Cross-Omics Comparison of Stress Responses in Mesothelial Cells Exposed to Heat- versus Filter-Sterilized Peritoneal Dialysis Fluids
}

\author{
Klaus Kratochwill, ${ }^{1,2}$ Thorsten O. Bender, ${ }^{1,3}$ Anton M. Lichtenauer, ${ }^{1,2}$ Rebecca Herzog, ${ }^{1,2}$ \\ Silvia Tarantino, ${ }^{1}$ Katarzyna Bialas, ${ }^{2}$ Achim Jörres, ${ }^{3}$ and Christoph Aufricht ${ }^{1}$ \\ ${ }^{1}$ Department of Pediatrics and Adolescent Medicine, Medical University of Vienna, 1090 Vienna, Austria \\ ${ }^{2}$ Zytoprotec GmbH, 1090 Vienna, Austria \\ ${ }^{3}$ Department of Nephrology and Medical Intensive Care, Campus Virchow-Klinikum, Charité Universitätsmedizin Berlin, \\ 13353 Berlin, Germany
}

Correspondence should be addressed to Christoph Aufricht; christoph.aufricht@meduniwien.ac.at

Received 13 May 2015; Accepted 31 August 2015

Academic Editor: Robert Beelen

Copyright (C) 2015 Klaus Kratochwill et al. This is an open access article distributed under the Creative Commons Attribution License, which permits unrestricted use, distribution, and reproduction in any medium, provided the original work is properly cited.

Recent research suggests that cytoprotective responses, such as expression of heat-shock proteins, might be inadequately induced in mesothelial cells by heat-sterilized peritoneal dialysis (PD) fluids. This study compares transcriptome data and multiple protein expression profiles for providing new insight into regulatory mechanisms. Two-dimensional difference gel electrophoresis (2DDIGE) based proteomics and topic defined gene expression microarray-based transcriptomics techniques were used to evaluate stress responses in human omental peritoneal mesothelial cells in response to heat- or filter-sterilized PD fluids. Data from selected heat-shock proteins were validated by 2D western-blot analysis. Comparison of proteomics and transcriptomics data discriminated differentially regulated protein abundance into groups depending on correlating or noncorrelating transcripts. Inadequate abundance of several heat-shock proteins following exposure to heat-sterilized PD fluids is not reflected on the mRNA level indicating interference beyond transcriptional regulation. For the first time, this study describes evidence for posttranscriptional inadequacy of heat-shock protein expression by heat-sterilized PD fluids as a novel cytotoxic property. Crossomics technologies introduce a novel way of understanding PDF bioincompatibility and searching for new interventions to reestablish adequate cytoprotective responses.

\section{Introduction}

Peritoneal dialysis (PD) is a cost effective and safe form of renal replacement therapy in end stage renal disease. However, PD-fluids (PDF) are bioincompatible solutions and may induce severe peritoneal damage, to a large part mediated by cytotoxic injury to the mesothelial cell layer, mostly due to low $\mathrm{pH}$, lactate, high glucose, and its degradation products $[1,2]$.

In experimental PD we and others have shown that acute exposure to cytotoxic contents of PDF results in rapid induction of heat shock proteins (HSP) in mesothelial cells during the recovery phase, counteracting toxic injury [3-6]. HSP are the most prominent protein members of the cellular stress response and transient overexpression of these important molecules of the cellular repair machinery has been shown to mediate strong cytoprotective effects during experimental PD $[5,6]$.

Recently, we have described unexpectedly low HSP expression upon more extended exposure to diluted heatsterilized PDF [7]. Albeit this setting still represents a highly artificial system, the exposure to diluted cytotoxic properties of PDF likely reflects intraperitoneal conditions during a PD dwell more closely than acute exposure to pure PDF [8-10]. Heat-sterilization and storage of glucose-based PDF result in formation of highly reactive glucose degradation 
products (GDPs) that are known to mediate their cytotoxicity via oxidative stress [11-13]. These findings suggest that exposure to PDF containing high levels of GDP may even dampen cellular stress responses, increasing the vulnerability of mesothelial cells against PDF cytotoxicity. Recent research suggests that oxidative stress might indeed suppress the cellular stress responses [14, 15].

In this study we have used two-dimensional difference gel electrophoresis (2D-DIGE) based proteomics and topic defined gene expression microarray-based transcriptomics techniques to evaluate mesothelial stress responses in response to exposure to heat- versus filter-sterilized PDF, thus comparing effects of GDPs on a global level. For the first time transcriptome data and multiple protein expression profiles were compared in experimental PD in order to provide new insight into regulatory mechanisms.

\section{Materials and Methods}

2.1. Materials. All chemicals, unless otherwise stated, were purchased from Sigma-Aldrich (St. Louis, MI, USA). All tissue culture plastics were Falcon (Becton Dickinson, San José, CA, USA). The PD solutions heat-sterilized PDF (H-PDF), containing GDPs, and filter-sterilized PDF (F$\mathrm{PDF}$ ), containing no GDPs, were prepared in the laboratory according to the following formulation: $\mathrm{NaCl} 5.786 \mathrm{~g} / \mathrm{L}$, $\mathrm{CaCl}_{2} \cdot 2 \mathrm{H}_{2} \mathrm{O} 0.257 \mathrm{~g} / \mathrm{L}, \mathrm{MgCl}_{2} \cdot 6 \mathrm{H}_{2} \mathrm{O} 0.102 \mathrm{~g} / \mathrm{L}$, sodium $\mathrm{D} / \mathrm{L}-$ lactate $3.925 \mathrm{~g} / \mathrm{L}$, and anhydrous $\mathrm{D}$-glucose 15.0 or $42.5 \mathrm{~g} / \mathrm{L}$, with a final composition in mmol/L: $\mathrm{Na}^{+} 132, \mathrm{Ca}^{2+} 1.25, \mathrm{Mg}^{2+}$ $0.25, \mathrm{Cl}^{-}$95, lactate 40 , and $3.86 \%$ glucose and a $\mathrm{pH}$ of 5.5 . The solutions from the same stock were then sterilized either by heat $\left(121^{\circ} \mathrm{C}, 0.2 \mathrm{MPa}, 20 \mathrm{~min}\right)$ or by filtration through a $0.2-\mu \mathrm{m}$ pore size filter (Nalgene, Nalge Nunc International, Rochester, NY, USA).

2.2. Exposure of Human Peritoneal Mesothelial Cells to PD Solutions. Human peritoneal mesothelial cells (HPMC) were isolated from fully anonymized specimens of omentum obtained from three consenting nonuremic patients undergoing elective abdominal surgery. The study was accomplished in accordance with the institutional review board, consistent with the principles of the Declaration of Helsinki. Cells were isolated and characterized as previously described [16]. HPMC were propagated in M199 culture medium supplemented with $2 \mathrm{mM}$ L-glutamine, $100 \mathrm{U} / \mathrm{mL}$ penicillin, $100 \mu \mathrm{g} / \mathrm{mL}$ streptomycin, $0.4 \mu \mathrm{g} / \mathrm{mL}$ hydrocortisone, and $10 \% \%_{v / v}$ fetal calf serum (FCS; Invitrogen, Carlsbad, CA, USA). All experiments were performed using cells from the second passage since later subcultures may contain an increasing number of senescent cells [16, 17]. HPMC were plated into multiwell clusters and grown until confluence. The standard medium containing $10 \%$ FCS was replaced by medium supplemented with $0.3 \%$ FCS for 48 hours prior to experiments to render the cells in a quiescent state.

PDF Exposure. In three independent experiments HPMC cultures obtained from the three above mentioned donors were exposed to a 1:1 mixture of regular culture medium containing $0.6 \%$ FCS (final concentration 0.3\%) and the mentioned PD solution (either filter- or heat-sterilized) for $24 \mathrm{~h}$. At the end of the exposure period the cells were harvested according to the procedure given below for $2 \mathrm{D}$ DIGE and the supernatants were saved at $-80^{\circ} \mathrm{C}$ until use in the viability assay (LDH release).

\subsection{Protein Expression Profiling 2D-DIGE}

2.3.1. Protein Sample Preparation. The cells were lysed by incubation with $1 \mathrm{~mL}$ lysis buffer (30 mM Tris, pH 8.5, $7 \mathrm{M}$ urea, $2 \mathrm{M}$ thiourea, 4\% 3-[(3-cholamidopropyl) dimethylammonio]-1-propanesulfonate (CHAPS), $1 \mathrm{mM}$ EDTA, 1 tablet of Complete Protease Inhibitor (Roche, Basel; Switzerland) per $100 \mathrm{~mL}$, and $10 \mu \mathrm{L} / \mathrm{mL}$ of each of the phosphatase inhibitor cocktails 1 and 2 (Sigma-Aldrich)) per $3 \times 10^{7}$ cells for $10 \mathrm{~min}$ at $25^{\circ} \mathrm{C}$. The resulting lysates were centrifuged for 30 minutes $\left(14,000 \times \mathrm{g}, 4^{\circ} \mathrm{C}\right)$ and stored at $-80^{\circ} \mathrm{C}$ until further processing. Total protein concentration was determined by the 2D-Quant Kit (GE Healthcare, Uppsala, Sweden) according to the manufacturer's manual.

2.3.2. Cell Harvesting and Protein Labeling. An internal pooled standard (IPS) containing all sample pools was prepared and used in all gels. IPS therefore represents a mixture of all proteins expressed in any cell under all tested conditions and should thus contain every protein spot that can be detected. Aliquots of the samples (H-PDF, F-PDF) were each labeled with Cy5 as well as protein lysates from immortalized HPMC, which were used as reference material. Labeling of the IPS was performed with Cy3 dye using the DIGE minimal labeling kit (GE Healthcare) following the recommendations of the manufacturer with minor modifications. In brief, $40 \mu \mathrm{g}$ of total protein per sample was mixed with $200 \mathrm{pmol}$ of the reconstituted Cy5 dye solution $(400 \mu \mathrm{M}$ stock solution in anhydrous DMF) and per gel $40 \mu \mathrm{g}$ of total protein of the internal pooled standard (IPS) of all samples was mixed with 200 pmol of the reconstituted Cy3 CyDye solution. Labeling of the IPS was performed in one batch to achieve a uniform standard. The labeling reactions were incubated on ice in the dark for $30 \mathrm{~min}$ and then stopped with $1 \mu \mathrm{L}$ of $10 \mathrm{mM}$ L-lysine solution. For every gel one Cy5 labeled sample and an aliquot of the Cy3 labeled IPS were mixed.

2.4. Isoelectric Focusing. The rehydration mix was brought to a final volume of $450 \mu \mathrm{L}$ with rehydration buffer consisting of $5 \mathrm{M}$ urea, $0.5 \%$ CHAPS, $0.5 \%$ Pharmalyte, and $12 \mu \mathrm{L} / \mathrm{mL}$ of DeStreak reagent (GE Healthcare). Each mixture was applied by rehydration loading to one IPG strip (ReadyStrip $\mathrm{pH} 3-$ 10, nonlinear, $24 \mathrm{~cm}$, Bio-Rad, Hercules, CA, USA) in the focusing tray of a Bio-Rad Protean IEF unit, sealed with silicone oil (Bio-Rad). The strips were rehydrated with the samples by "active rehydration" at $50 \mathrm{~V}$ and $20^{\circ} \mathrm{C}$ for $15 \mathrm{~h}$ and then focused for $3 \mathrm{~h}$ at $100 \mathrm{~V}$, before the voltage was constantly increased to $8000 \mathrm{~V}$ within 17.5 hours, applying altogether $65 \mathrm{kVh}$ with a maximum of $30 \mu \mathrm{A}$ per strip.

2.5. Vertical Electrophoresis. Gels for second-dimension vertical SDS-PAGE were cast using a Bio-Rad multicasting 
chamber, low-fluorescent glass plates, and $1 \mathrm{~mm}$ spacers (BioRad). For a final concentration of the separation gels of $12 \%, 240 \mathrm{~mL}$ acrylamide stock solution (40\%, T : C = 29:1, Bio-Rad) was mixed with $200 \mathrm{~mL} 1.5 \mathrm{M}$ Tris- $\mathrm{HCl}$ pH 8.8, $40 \mathrm{~mL}$ glycerol, and $320 \mathrm{~mL} \mathrm{H}_{2} \mathrm{O}_{\mathrm{UHQ}}$. TEMED $(80 \mu \mathrm{L})$ and ammonium persulfate ( $1 \mathrm{~mL}$, APS, $10 \%$ in $\mathrm{H}_{2} \mathrm{O}_{\mathrm{UHQ}}$ ) were added after degassing of the mixture and right before filling of the casting chamber. The gels were left to polymerize overnight, overlaid with water-saturated n-butanol. Vertical second-dimension SDS-PAGE was carried out on a Bio-Rad Dodeca system with the current set to $60 \mathrm{~mA}$ for $100 \mathrm{Vh}$ and then to $200 \mathrm{~mA}$ for $1200 \mathrm{Vh}$.

2.6. Fluorescence Image Acquisition and Data Analysis. DIGE labeled gels were scanned sandwiched between the lowfluorescent glass plates of the cassettes immediately after the run. Gel images were acquired using a Typhoon Trio laser scanner (GE Healthcare) using excitation and emission wavelengths recommended for the used dyes (Cy3: Ex $532 \mathrm{~nm}$, Em 580 nm, and BP 30; Cy5: Ex 633 nm, Em 670 nm, and BP 30). The photomultiplier voltage was chosen so that the most abundant protein spots were close to saturation. Sensitivity level was set to "normal."

Gel images were analyzed using the Delta2D 3.6 software (Decodon $\mathrm{GmbH}$, Greifswald, Germany) using the algorithm designated for DIGE experiments. The images, containing the IPS, were aligned by pairwise warping and spot detection was carried out on a fused image of all gels (see Supplemental Figure 1 in Supplementary Material available online at http://dx.doi.org/10.1155/2015/628158). Protein identifications, accomplished in our laboratory [18], were processed with the aid of the ID mapping feature offered by the UniProt database (http://www.uniprot.org; [19]) to a short list of proteins overlapping with the genes investigated by RNA array used in this study (see Supplemental Table 1 for a summary of all used mass spectrometric identification data). The protein annotations of this short list were assigned to the respective spots on the $2 \mathrm{D}$ gels. Relative spot volumes normalized to the IPS of 28 unique proteins (see Table 1) were quantified among exposure of HPMC to H-PDF or F-PDF. Significance values were derived from group comparisons utilizing Student's $t$-test with the obtained $p$ values given in Table 1. Details on individual proteins and corresponding spots are provided as bar graphs for each spot (Supplemental Figure 2) and spot album (Supplemental Figure 3).

2.7. RNA Expression Array Analysis. For analysis on the transcriptional level topic defined microarray experiments were employed. In brief, HPMC exposed to H-PDF or F-PDF were homogenized in $350 \mu \mathrm{L}$ RLT buffer (Qiagen, Hilden, Germany) and then extracted using the RNeasy Mini Kit (Qiagen) according to the manufacturer's protocol. Total RNA was checked for integrity with the Agilent Bioanalyzer 2100 (Agilent Technologies, Inc., Palo Alto, CA). $0.8 \mu \mathrm{g}$ of total RNA was then used for amplification and analysis with topic defined PIQOR Toxicology Human Microarray (Miltenyi Biotec, Bergisch Gladbach, Germany) containing 1264 human genes comprising the subject areas apoptosis,
DNA damage and repair, inflammation, cell proliferation and response to oxidative stress, and xenobiotic metabolism. Each PIQOR microarray contains six housekeeping genes (ACTA2, CYPA, GAPDH, HPRT, TUBA, and TUBB) and six controls (herring sperm DNA, salt, and four artificial control RNAs) for the correct quantification of the differential expression patterns. Genes are spotted in quadruplicate. All steps between RNA isolation and data interpretation, including sample labelling, microarray hybridization, and scanning, were carried out by Miltenyi Biotec Microarray Services.

Data analysis included exclusion of low-quality spots, background subtraction to obtain the net signal intensity, data normalization, and calculation of the $\mathrm{Cy} 5 / \mathrm{Cy} 3$ ratios. Additionally only spots that had at least in one channel a signal intensity that was 2-fold higher than the mean background were taken into account for the ratio calculation. Normalized mean Cy5/Cy3 ratios of the four replicates per gene and the respective coefficient of variation (\% CV) were calculated. This CV refers to the average of the $\mathrm{Cy} 5 / \mathrm{Cy} 3$ ratios for the gene replicates.

Finally, for the genes overlapping with identified proteins in the proteomics experiment mean ratios were calculated by averaging the values obtained from the three individual chips, one per biological experiment. The standard deviation (SD) and $\mathrm{CV}$ (Chip CV) are also given in the results (see Table 1).

2.8. Two-Dimensional Western Blotting (Adapted from [20]). For 2D western analysis, gels were prepared as described above. Proteins were electroblotted onto PVDF membranes (Millipore; Billerica, MA, USA) immediately after the run by semidry transfer using the Novablot unit of the MultiPhor II electrophoresis system and an according transfer buffer (200 mM glycine, $25 \mathrm{mM}$ Tris base, $0.1 \%$ SDS, and $20 \%$ methanol). The membranes were washed in TBST buffer (150 mM NaCl, 0.05\% Tween 20, and $10 \mathrm{mM}$ Tris- $\mathrm{HCl}$ at $\mathrm{pH}$ 7.4) and stained using ruthenium II tris-bathophenanthroline disulfonate (RuBPS) following a fluorescent staining protocol, modified for staining membranes. In brief, RuBPS was prepared as published by Rabilloud et al. [21] and used as stock solution without further processing. The proteins were fixed by incubation for $15 \mathrm{~min}$ with fixing solution (10\% acetic acid, $20 \%$ methanol). Membranes were washed 4 times for 5 min with $\mathrm{H}_{2} \mathrm{O}_{\mathrm{UHQ}}$ and then incubated for 30 min with staining solution $(10 \mu \mathrm{L}$ RuBPS stock solution made up to $1000 \mathrm{~mL}$ with $\mathrm{H}_{2} \mathrm{O}_{\mathrm{UHQ}}$ ). After again washing 4 times for $5 \mathrm{~min}$ with $\mathrm{H}_{2} \mathrm{O}_{\mathrm{UHQ}}$ the membranes were dried and scanned with the aid of the Typhoon Trio laser scanner mentioned above using excitation and emission wavelengths optimized for the used protocol (Ex $488 \mathrm{~nm}$, Em $670 \mathrm{~nm}$, and BP 30). The photomultiplier voltage was chosen so that the stained protein spots were clearly distinguishable from the background. Sensitivity level was set to "normal." The obtained total protein pattern was used for later alignment to the specific immunodetected signals. The membranes were rehumidified with methanol and washed again in TBST buffer before proceeding to the blocking step. The membranes were blocked with 5\% dry milk in TBST and then incubated 


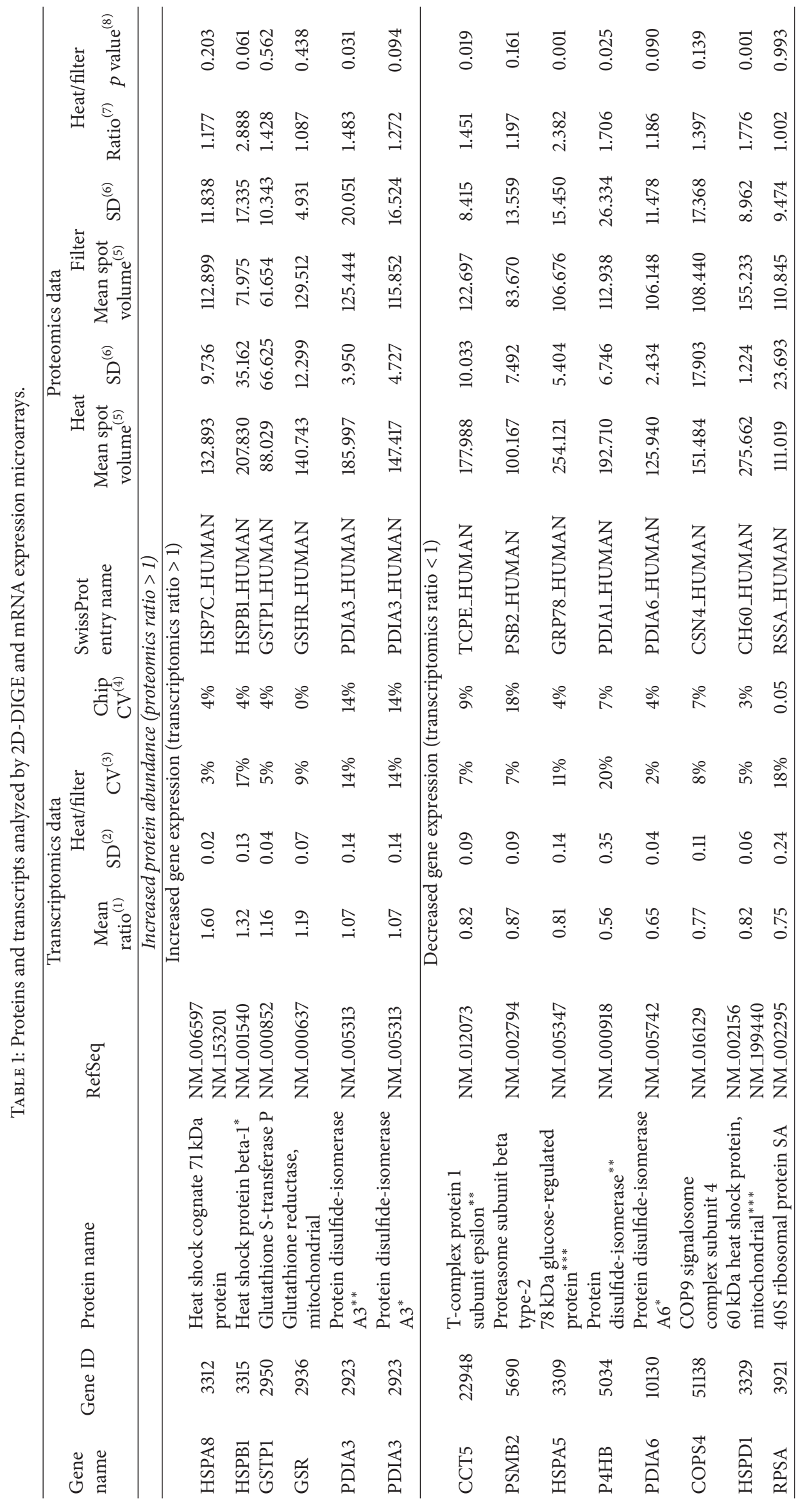




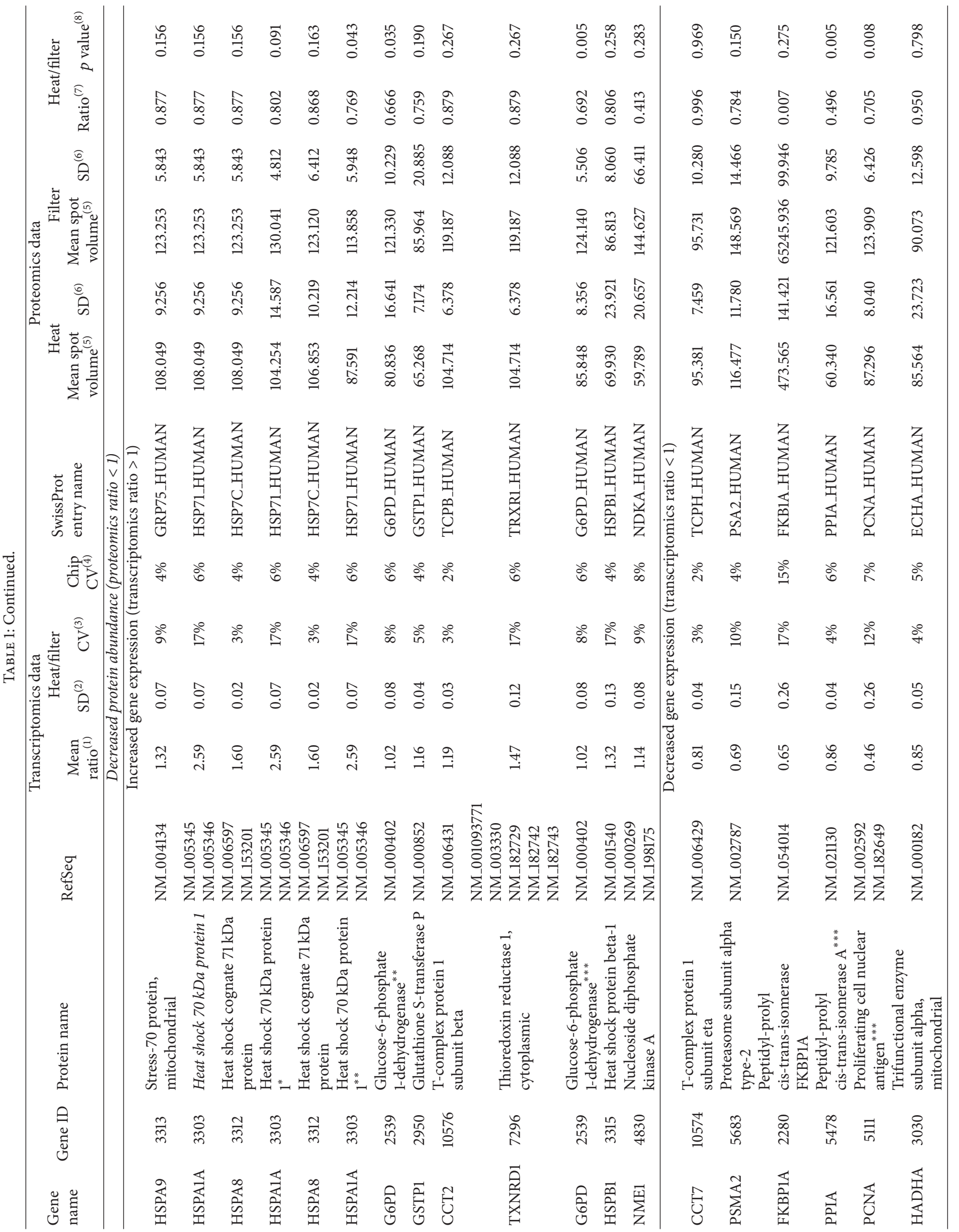




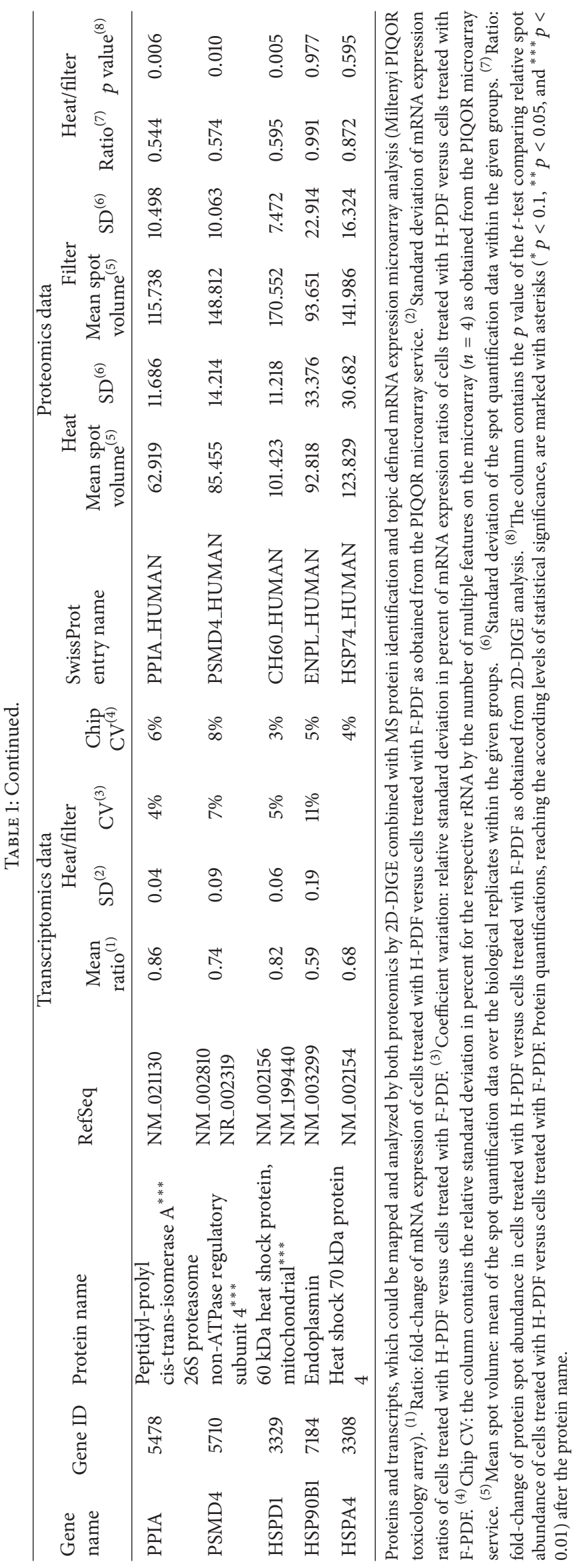


with the primary murine antibody against Hsp72 (SPA-810, Stressgen/Assay Designs, Ann Arbor, MI, USA), Hsp27 (SPA801, Stressgen/Assay Designs), or Hsp60 (SPA-806 Stressgen/Assay designs) dissolved in TBST containing $1 \%$ dry milk for 6 hours. After washing 3 times for $20 \mathrm{~min}$ in TBST and incubation with a secondary, peroxidase-coupled antibody (polyclonal rabbit anti-mouse Ig/HRP P0260, Dako Cytomation, Carpinteria, CA, USA) detection was accomplished by using enhanced chemiluminescence solution (Western Lightning Reagent, Perkin Elmer, Boston, MA, USA) and a ChemiDoc XRS chemiluminescence detection system (BioRad).

\section{Results}

Exposure to H-PDF and F-PDF resulted in sublethal injury, evaluated by indiscernible cell density, assessed by light microscopy and cell counting, and comparable total protein concentrations of the cell lysates (H-PDF/F-PDF 76.6 $236.7 \%$ mean $\pm \mathrm{SD}, p=0.338)$. $\mathrm{LDH}$ release as a marker of loss of cellular membrane integrity was significantly higher following exposure to H-PDF than to F-PDF (H-PDF/F-PDF $887 \pm 277 \%$ mean \pm SD, $p=0.011$ ).

For investigating potentially involved regulatory mechanisms both protein and RNA levels of HPMC undergoing treatment with H-PDF or F-PDF for $24 \mathrm{~h}$ were analyzed by 2D-DIGE and topic defined gene expression microarrays. In order to cover the largest possible number of transcripts and proteins we used all available mass spectrometric protein identifications in MC performed by our group until today $[18,22]$ and built a comprehensive $2 \mathrm{D}$ proteome map (see Supplemental Figure 1 and Supplemental Table 1). These identifications were screened for overlaps with the transcripts successfully quantified in the mRNA microarray. Of the 1264 genes contained on the microarray and the overall 185 protein identifications in mesothelial cells by mass spectrometry we could identify an overlap of 28 unique genes with the according proteins contained in 38 distinct spots (see Table 1 , Supplemental Figure 2, and Supplemental Figure 3). The high reproducibility of the proteomics data obtained by 2DDIGE was reflected by a low variability of the quantified spots (median CV was 9.7\% for all protein spots contained in Table 1). The observation of more than one spot per protein is explained by the capability of this technique to detect individual isoforms or posttranslationally modified variants of the same protein. Exploration of the combined RNA and protein profiles allowed functional grouping of the analyzed candidates according to observed regulation patterns.

When grouping these protein spots according to their expression on the protein and RNA level by calculation of a H-PDF/F-PDF ratio, four discrete groups could be built (see Figure 1). Thirteen spots had a protein ratio below 1.0 , meaning less protein abundance when exposed to $\mathrm{H}$ PDF, whereas their RNA ratio was above 1.0. Seven of these spots (54\%) contained HSP (protein symbols: HSPA9, HSPA1A, HSPA8, HSPA1A, HSPA8, HSPA1A, G6PD, GSTP1, CCT2, TXNRD1, G6PD, HSPB1, and NME1). Six protein spots showed a protein ratio above 1.0 with simultaneously elevated RNA expression (>1.0). Two of these spots contained
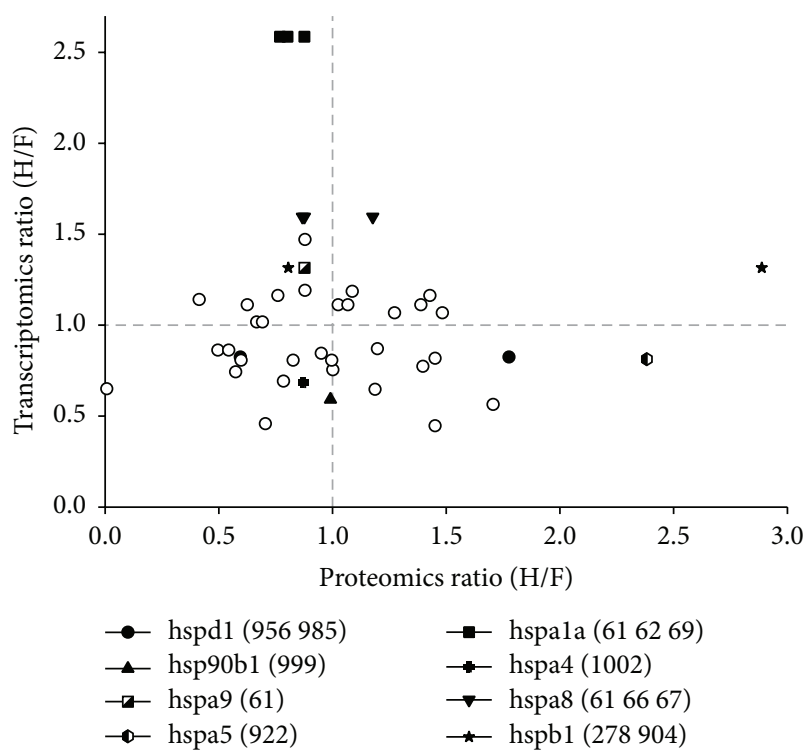

FIGURE 1: Cross-omics comparison of stress responses in mesothelial cells exposed to heat-versus filter-sterilized peritoneal dialysis fluid. HPMC cultures $(n=3)$ were continuously exposed to a 1:1 mix of heat- or filter-sterilized PDF ("H" or "F") and cell culture medium for 24 hours. Data are expressed as ratio of the respective proteomics and transcriptomics results from heat- over those of filter-sterilized PDF exposed mesothelial cells (H/F). The comparison of proteomics and transcriptomics data allowed the discrimination of differentially regulated protein expression into groups depending on correlating or noncorrelating transcripts. The inadequate expression of several HSP (full symbols) on the protein level is not reflected on the transcriptional level indicating potential interference of GDPs in translational activity and regulation.

HSP (HSPA8, HSPB1, GSTP1, GSR, PDIA3, and PDIA3). However, other protein isoforms of these two HSP (HSPA8, HSPB1) were also contained in the previously mentioned group. Eight protein spots showed higher abundance on the protein level although their RNA expression ratio was below 1.0. Two of these spots contained HSP (CCT5, PSMB2, HSPA5, P4HB, PDIA6, COPS4, HSPD1, and RPSA). Eleven protein spots had a H-PDF/F-PDF ratio of less than 1.0 with concomitantly downregulated RNA expression. Three of these spots contained HSP (CCT7, PSMA2, FKBP1A, PPIA, PCNA, HADHA, PPIA, PSMD4, HSPD1, HSP90B1, and HSPA4). In addition to the graphical presentation in Figure 1, numerical values of RNA expression ratios as well as spot abundance data under the experimental conditions of extended heat- versus filter-sterilized PDF treatment are given in Table 1 together with their statistical parameters and $p$ values indicating significant changes. Additional bioinformatics analysis of the transcriptomics data only found enrichment of biological processes attributable to immune response, angiogenesis, injury/repair mechanism, and apoptosis (see Supplemental Table 2).

In Figure 2, results of 2D western blotting are shown for the protein spots of prototypical members of the major HSP families, that is, Hsp72 (HSPA1A), Hsp27 (HSPB1), and Hsp60 (HSPD1), demonstrating that the mass spectrometric 

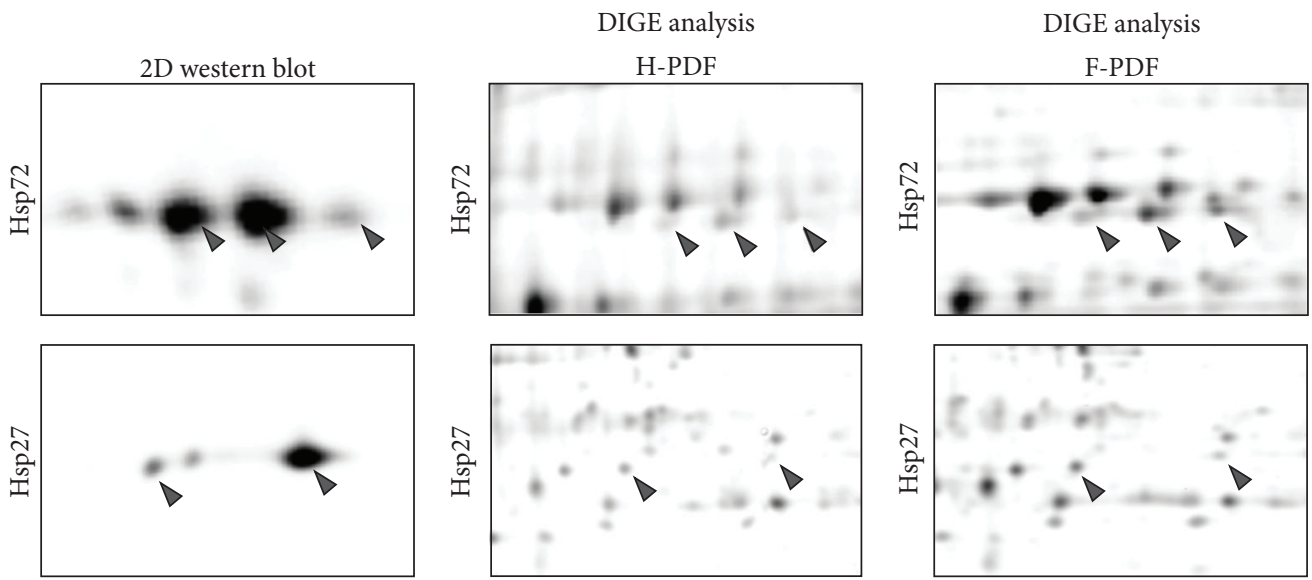

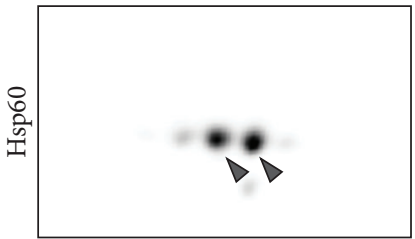

(a)

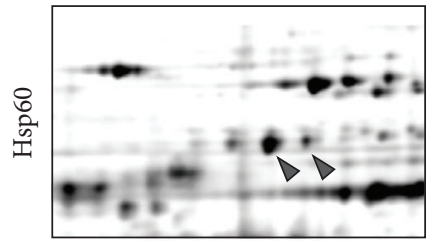

(b)

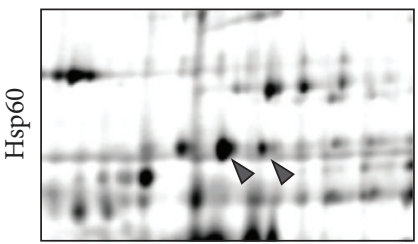

(c)

Figure 2: Two-dimensional western analysis of the chaperones Hsp70, Hsp27, and Hsp60. (a) shows the result of the immunoblots with positive signals for the respective specific antibodies given in each line and the MS identified protein spots indicated by grey arrow heads. For immunodetection of all relevant spots, pooled samples were used in order to represent all relevant isoforms and modifications. (b, c) show the identical regions in the DIGE gels, where the middle panel shows the protein separation of total cell extracts from mesothelial cells following exposure to H-PDF, and (c) shows the protein separation of total cell extracts from mesothelial cells following exposure to F-PDF. Again the MS identified protein spots are indicated by grey arrow heads.

data on protein identities might not cover all isoforms that are recognized by specific antibodies. Whereas the antibodybased detection might yield unspecific signals, the spot identities that were confirmed by both western blotting and mass spectrometric data represent very robust information.

\section{Discussion}

Comparing the effects of two PDF that only differ in their modality of sterilization, either by heat or by filtration, can be regarded as surrogate method to evaluate the effects of GDPs formed through heat-sterilization [23]. Specific effects of heat-sterilized PDF on the cellular stress response might thus be largely attributed to these toxic compounds. GDPs are known to mediate their cytotoxicity via oxidative stress, and recent research suggests that oxidative stress might dampen the cellular stress responses $[14,15]$.

The advent of omics technologies, such as gel- or mass spectrometry-based proteomics of the protein level and microarray techniques on the transcriptional level, allowed unbiased global analyses, searching for yet unknown differentially regulated proteins or transcripts under given experimental conditions.

Whereas the use of a single analytical level allows candidate search and functional interpretation based on common features, for example, characterized in gene ontology databases, the cross-omics approach allows generating hypotheses by comparing gene expression profiling to (functional active) protein abundances. In this study we used a topic defined mRNA microarray for toxicology relevant genes, provided by Miltenyi as the PIQOR platform. In contrast to quantification of mRNA levels by rt-PCR, which relies on the consistency of single housekeeping genes, the array technique allows more elaborate approaches of normalization.

Together with our proteomics platform based on 2DDIGE we compared effects of heat- versus filter-sterilized PDF in mesothelial cells in the extended exposure model, for proteins identified by mass spectrometry in earlier in vitro $\mathrm{PD}$ studies.

The high relevance of the chosen candidate proteins and transcripts becomes evident by the considerable overlap of MS identified proteins and genes represented on the PIQOR array in experimental PD. We could therefore use this set, mainly consisting of chaperones and stress-relevant proteins involved in detoxification and protein homeostasis, to search for global systematic effects of heat- versus filter-sterilized PDF on the cellular stress response. In recent work, we have shown at the protein level that expression levels of such a stress proteome can be related to mesothelial cell susceptibility to PDF induced injury [24]. This stress proteome was found to be downregulated following exposure to heatsterilized PDF and could be restored by addition of cytoprotective additives, such as alanyl-glutamine dipeptide [24]. The cross-omics approach used in the current study gives additional information beyond the observed inadequate induction of the heat shock response at the protein level $[7,24]$. 
In our dataset a rather large group of candidates showed a protein abundance ratio below 1.0 (downregulated by $\mathrm{H}$ PDF compared to F-PDF) and at the same time an mRNA expression ratio above 1.0 (upregulated by $\mathrm{H}$-PDF compared to F-PDF), when the two fluid types were compared. This phenomenon is of growing interest in the omics-field, as the observed perturbations might allow deducing regulatory mechanisms [25]. Future studies are needed to investigate whether the reverse relationship of protein and mRNA indicates a higher degree of protein turnover (i.e., shortened halflife of the protein by increased degradation) or translational inhibition or posttranslational modifications [26, 27]. This group contains many HSP but the effect only reached the level of significance for the proteins Hsp72 (HSPA1A) and glucose-6-phosphate dehydrogenase (G6PD), which might be due to inherent limited power of these hypothesesgenerating omics studies. HSP are molecular chaperones and known to incorporate transport and folding of other proteins by binding to hydrophobic normal hidden domains of immature or denatured proteins. Thereby, these proteins have been shown to promote cytoskeletal repair and preserve the mesothelial monolayer [5, 6]. Whereas G6PD was already found upregulated in the acute exposure model in an earlier study [28] this key-enzyme of the pentose phosphate pathway, which is substantial for the production of NADPH and the cell's resistance to oxidative stress $[29,30]$, is significantly downregulated by heat-sterilized PDF compared to filtersterilized PDF after extended exposure. The initial observation that the cells are not able to adequately respond to stress mediated by toxic factors of PDF, such as glucose and GDPs, is further reflected by the fact that all candidates in the dataset belonging to the glutathione system (GSTP1, TXNRD1, and GSR), which is dedicated to detoxification and neutralization of reactive oxygen species, are found to be upregulated on the transcriptional level. However, none of these candidates show significant upregulation of protein abundance. The deleterious effect of the cooccurrence of hyperglycemia, toxic aldehydes, such as GDPs, and a lack of reductive power (e.g., by NADPH and glutathione) has been demonstrated extensively in the diabetes model and beyond [31-33].

Two further subgroups of our dataset were characterized by either simultaneously increased or decreased abundance of both protein and mRNA. Concurrent regulation of protein and mRNA likely indicates undisturbed translation of transcriptional regulation of gene expression into proteins.

One clearly upregulated player identified in our study is PDIA3, a protein disulfide-isomerase predominantly found in the endoplasmic reticulum lumen, which is involved in the unfolded protein response [34]. Interestingly, knockdown of PDIA3, also known as endoplasmic reticulum resident protein 57 or $58 \mathrm{kDa}$ glucose-regulated protein, protected against tunicamycin-induced apoptosis, with associated induction of the $78 \mathrm{kDa}$ glucose-regulated protein (GRP-78, BiP, and HSPA5) [35], which was identified as downregulated on the transcriptional level but upregulated on the protein level in our study. GRP-78 is an ER stress protein (glucose-regulated protein) and, as suggested by its name, expected to be upregulated by the physicochemical properties of PDF.
Another protein found with significantly higher abundance, when H-PDF was compared to F-PDF, was Hsp27 (HSPB1) which is well known to be involved in the stress response to $\mathrm{PDF}[3,4]$. Hsp27 is a highly abundant effector of the heat shock response directly interacting with the actin cytoskeleton and thereby protecting mesothelial cell integrity by increased abundance [36]. However, it is well known that Hsp27 is extensively modified by phosphorylation leading to reciprocal abundance changes of multiple spots on 2D gels $[36,37]$. Indeed we also identified Hsp27 in a spot with lowered abundance, as can be better made evident by direct comparison between $2 \mathrm{D}$ proteomic gels and $2 \mathrm{D}$ western analysis. As the phosphorylation status is functionally highly relevant for its chaperoning effect, Hsp27 is a particularly informative candidate to demonstrate the role of posttranslational modification on the level of functionally active protein isoforms versus total protein abundance and gene expression.

One clearly downregulated player, on the protein level as well as on the transcriptional level, identified in our study is PPIA which is a peptidyl-prolyl cis-trans-isomerase and is thereby active in protein folding [38]. PPIA is also a member of the immunophilin family (cyclophilin A) [39] and the receptor for the immunosuppressive drugs tacrolimus and cyclosporine [40, 41]. Other examples for proteins with significantly downregulated transcripts were the important ER chaperone Hsp60 (HSPD1), which was as Hsp27 also found in a significantly upregulated spot, and the proliferating cell nuclear antigen (PCNA), which is also known as cyclin, reflecting an increase in MC proliferation as previously described in the in vivo setting [42].

A small group of candidates showed a protein abundance ratio above 1.0 (upregulated by H-PDF compared to F-PDF) and at the same time an mRNA expression ratio below 1.0 (downregulated by H-PDF compared to F-PDF), when the two fluid types were compared. In this case the increased levels of protein expression with concomitantly depressed transcription of the same genes allow generating interesting hypotheses, such as prolonged half-life of the proteins by decreased degradation, possibly mediated by lack of protein degrading mechanisms such as the ubiquitin-proteasome pathway [26].

Interestingly all identified members associated with the ubiquitin-proteasome pathway (PSMB2, COPS4, PSMA2, and PSMD4) showed downregulated levels of mRNA and one of them was also significantly lower abundant on the protein level. This effect is concordant with the literature, where hyperglycemia and methylglyoxal led to an impaired ubiquitin-proteasome pathway in bovine and murine endothelial cells [32]. Indeed methylglyoxal is one of the GDPs detected in considerable concentrations in conventional PDF [43]. In this study nonuremic patients were used to obtain primary mesothelial cells from omentum. It has been demonstrated before that the uremic milieu per se can change the behavior of the mesothelial cells. Thus, future studies are needed to investigate whether specific GDPs in PDF might impair or deplete this essential part of the cellular stress response and what the role of the uremic milieu with even more toxic small molecules might be $[44,45]$. 
Our data show that under stressful conditions the correlation between mRNA and protein cannot be regarded as linear for a wide range of tightly involved players of the stress response. The transcription of mRNA is the initial level of gene regulation, where transcription factors lead to situation-dependent usage of genetic sequence. However a plethora of intermitting mechanisms, such as RNA interference, regulatory proteins, or translational efficiency, can promote or hinder cellular protein production. Finally the amounts of functional proteins are influenced by protein folding, posttranslational modification, and turnover [26, 27]. Albeit the observed changes on the protein level are quite low, they are in a reasonable biological range, given that the proteins that can be detected by $2 \mathrm{D}$ gel electrophoresis represent the most abundant portion of soluble proteins. These findings highlight the limitations of gene expression profiling concerning the prediction of abundance of functionally active proteins and/or their isoforms. Future studies need to carefully assess these regulatory mechanisms to monitor the abundance of effector proteins that ultimately reflect biological reality.

Bioinformatics analysis yields similar information on activated stress responses using data derived from this transcriptomics dataset as we have previously reported in a proteomics approach [22]. Direct comparison between these "omics" technologies at the level of individual gene products, however, as we performed in our cross-omics approach, might allow additional interesting insights into specific pathogenic processes caused by PDF exposure. Moreover, the results of the current study underline that information of potential diagnostic (such as biomarker candidates) and/or therapeutic (such as novel drug targets) implications derived from proteomics and/or transcriptomics findings cannot be utilized interchangeably but rather request specific separate analysis and interpretation.

Taken together, the comparison of proteomics and transcriptomics data allowed the discrimination of differentially regulated protein expression according to correlating or noncorrelating transcripts. The results of this study are particularly interesting in terms of limitations of gene expression profiling with regard to prediction of abundance of functionally active proteins, indicating the need for future studies to investigate potential interference in translational activity and regulation.

\section{Conflict of Interests}

Klaus Kratochwill, Anton M. Lichtenauer, Rebecca Herzog, and Katarzyna Bialas are employees of Zytoprotec GmbH. Christoph Aufricht is cofounder of Zytoprotec $\mathrm{GmbH}$, a spin-off of the Medical University of Vienna that holds the patent "Carbohydrate-Based Peritoneal Dialysis Fluid Comprising Glutamine Residue" (International Publication no. WO 2008/106702 A1). Thorsten O. Bender is a recipient of an EMBO (ERA-EDTA) stipend. Achim Jörres is consultant to Fresenius Medical Care.

\section{Authors' Contribution}

Klaus Kratochwill and Thorsten O. Bender contributed equally to this work.

\section{Acknowledgments}

This study was funded by the ZIT, the Technology Agency of the City of Vienna (ID 648625). Klaus Kratochwill, Silvia Tarantino, Katarzyna Bialas, Achim Jörres, and Christoph Aufricht were supported by the European Training and Research in Peritoneal Dialysis (EuTRiPD) program, a project funded by the European Union within the Marie Curie scheme (287813). The authors are grateful for the technical support by Astrid Scherr and Axana Hellmann in preparing the paper and figures.

\section{References}

[1] O. Devuyst, N. Topley, and J. D. Williams, "Morphological and functional changes in the dialysed peritoneal cavity: impact of more biocompatible solutions," Nephrology Dialysis Transplantation, vol. 17, supplement 3, pp. 12-15, 2002.

[2] A. Jorres and J. Witowski, "Lessons from basic research for PD treatment," Peritoneal Dialysis International, vol. 25, supplement 3, pp. S35-S38, 2005.

[3] K. Arbeiter, B. Bidmon, M. Endemann et al., "Peritoneal dialysate fluid composition determines heat shock protein expression patterns in human mesothelial cells," Kidney International, vol. 60, no. 5, pp. 1930-1937, 2001.

[4] C. Aufricht, M. Endemann, B. Bidmon et al., "Peritoneal dialysis fluids induce the stress response in human mesothelial cells," Peritoneal Dialysis International, vol. 21, no. 1, pp. 85-88, 2001.

[5] B. Bidmon, M. Endemann, K. Arbeiter et al., "Overexpression of HSP-72 confers cytoprotection in experimental peritoneal dialysis," Kidney International, vol. 66, no. 6, pp. 2300-2307, 2004.

[6] M. Endemann, H. Bergmeister, B. Bidmon et al., "Evidence for HSP-mediated cytoskeletal stabilization in mesothelial cells during acute experimental peritoneal dialysis," American Journal of Physiology-Renal Physiology, vol. 292, no. 1, pp. F47-F56, 2007.

[7] T. O. Bender, M. Böhm, K. Kratochwill et al., "Peritoneal dialysis fluids can alter HSP expression in human peritoneal mesothelial cells," Nephrology Dialysis Transplantation, vol. 26, no. 3, pp. 1046-1052, 2011.

[8] N. Topley, "What is the ideal technique for testing the biocompatibility of peritoneal dialysis solutions?" Peritoneal Dialysis International, vol. 15, no. 6, pp. 205-209, 1995.

[9] X. L. Zhang, N. Topley, T. Ito, and A. Phillips, "Interleukin6 regulation of transforming growth factor (TGF) $\beta$ receptor compartmentalization and turnover enhances TGF- $\beta 1$ signaling," Journal of Biological Chemistry, vol. 280, no. 13, pp. 1223912245, 2005.

[10] C. J. Holmes, "Pre-clinical biocompatibility testing of peritoneal dialysis solutions," Peritoneal Dialysis International, vol. 20, supplement 5, pp. S5-S9, 2001.

[11] M. Erixon, T. Lindén, P. Kjellstrand et al., "PD fluids contain high concentrations of cytotoxic GDPs directly after sterilization," Peritoneal Dialysis International, vol. 24 , no. 4, pp. $392-$ 398, 2004. 
[12] M. Erixon, A. Wieslander, T. Lindén et al., "How to avoid glucose degradation products in peritoneal dialysis fluids," Peritoneal Dialysis International, vol. 26, no. 4, pp. 490-497, 2006.

[13] A. Jörres, T. O. Bender, and J. Witowski, "Glucose degradation products and the peritoneal mesothelium," Peritoneal Dialysis International, vol. 20, supplement 5, pp. S19-S22, 2001.

[14] P. L. Hooper and J. J. Hooper, "Loss of defense against stress: diabetes and heat shock proteins," Diabetes Technology and Therapeutics, vol. 7, no. 1, pp. 204-208, 2005.

[15] M. Adachi, Y. Liu, K. Fujii et al., "Oxidative stress impairs the heat stress response and delays unfolded protein recovery," PLoS ONE, vol. 4, no. 11, Article ID e7719, 2009.

[16] E. Stylianou, L. A. Jenner, M. Davies, G. A. Coles, and J. D. Williams, "Isolation, culture and characterization of human peritoneal mesothelial cells," Kidney International, vol. 37, no. 6, pp. 1563-1570, 1990.

[17] K. Ksiaz̧ek, K. Piwocka, A. Brzezińska et al., "Early loss of proliferative potential of human peritoneal mesothelial cells in culture: the role of p16INK4a-mediated premature senescence," Journal of Applied Physiology, vol. 100, no. 3, pp. 988-995, 2006.

[18] K. Kratochwill, M. Lechner, A. M. Lichtenauer et al., "Interleukin-1 receptor-mediated inflammation impairs the heat shock response of human mesothelial cells," The American Journal of Pathology, vol. 178, no. 4, pp. 1544-1555, 2011.

[19] The-UniProt-Consortium, "The universal protein resource (UniProt)," Nucleic Acids Research, vol. 36, pp. D190-D195, 2008.

[20] A. M. Lichtenauer, R. Herzog, S. Tarantino, C. Aufricht, and K. Kratochwill, "Equalizer technology followed by DIGE-based proteomics for detection of cellular proteins in artificial peritoneal dialysis effluents," Electrophoresis, vol. 35, no. 10, pp.13871394, 2014.

[21] T. Rabilloud, J.-M. Strub, S. Luche, A. van Dorsselaer, and J. Lunardi, "A comparison between Sypro Ruby and ruthenium II tris (bathophenanthroline disulfonate) as fluorescent stains for protein detection in gels," Proteomics, vol. 1, no. 5, pp. 699-704, 2001.

[22] K. Kratochwill, M. Lechner, C. Siehs et al., "Stress responses and conditioning effects in mesothelial cells exposed to peritoneal dialysis fluid," Journal of Proteome Research, vol. 8, no. 4, pp. 1731-1747, 2009.

[23] A. Wieslander, T. Linden, and P. Kjellstrand, "Glucose degradation products in peritoneal dialysis fluids: how they can be avoided," Peritoneal Dialysis International, vol. 21, 3, pp. S119S124, 2001.

[24] K. Kratochwill, M. Boehm, R. Herzog et al., "Alanyl-glutamine dipeptide restores the cytoprotective stress proteome of mesothelial cells exposed to peritoneal dialysis fluids," Nephrology Dialysis Transplantation, vol. 27, no. 3, pp. 937-946, 2012.

[25] S. P. Gygi, Y. Rochon, B. R. Franza, and R. Aebersold, "Correlation between protein and mRNA abundance in yeast," Molecular and Cellular Biology, vol. 19, no. 3, pp. 1720-1730, 1999.

[26] T. Maier, M. Güell, and L. Serrano, "Correlation of mRNA and protein in complex biological samples," FEBS Letters, vol. 583, no. 24, pp. 3966-3973, 2009.

[27] R. Herzog, T. O. Bender, A. Vychytil, K. Bialas, C. Aufricht, and K. Kratochwill, "Dynamic O-linked N-acetylglucosamine modification of proteins affects stress responses and survival of mesothelial cells exposed to peritoneal dialysis fluids," Journal of the American Society of Nephrology, vol. 25, no. 12, pp. 27782788, 2014.

[28] M. Lechner, K. Kratochwill, A. Lichtenauer et al., "A proteomic view on the role of glucose in peritoneal dialysis," Journal of Proteome Research, vol. 9, no. 5, pp. 2472-2479, 2010.

[29] M. V. Ursini, A. Parrella, G. Rosa, S. Salzano, and G. Martini, "Enhanced expression of glucose-6-phosphate dehydrogenase in human cells sustaining oxidative stress," Biochemical Journal, vol. 323, no. 3, pp. 801-806, 1997.

[30] W.-N. Tian, L. D. Braunstein, K. Apse et al., "Importance of glucose-6-phosphate dehydrogenase activity in cell death," The American Journal of Physiology-Cell Physiology, vol. 276, no. 5, pp. C1121-C1131, 1999.

[31] M. Brownlee, "Biochemistry and molecular cell biology of diabetic complications," Nature, vol. 414, no. 6865, pp. 813-820, 2001.

[32] M. A. Queisser, D. Yao, S. Geisler et al., "Hyperglycemia impairs proteasome function by methylglyoxal," Diabetes, vol. 59, no. 3, pp. 670-678, 2010.

[33] F. Salvemini, A. Franzé, A. Iervolino, S. Filosa, S. Salzano, and M. V. Ursini, "Enhanced glutathione levels and oxidoresistance mediated by increased glucose-6-phosphate dehydrogenase expression," The Journal of Biological Chemistry, vol. 274, no. 5, pp. 2750-2757, 1999.

[34] M. Ni and A. S. Lee, "ER chaperones in mammalian development and human diseases," FEBS Letters, vol. 581, no. 19, pp. 3641-3651, 2007.

[35] D. Xu, R. E. Perez, M. H. Rezaiekhaligh, M. Bourdi, and W. E. Truog, "Knockdown of ERp57 increases BiP/GRP78 induction and protects against hyperoxia and tunicamycininduced apoptosis," The American Journal of Physiology-Lung Cellular and Molecular Physiology, vol. 297, no. 1, pp. L44-L51, 2009.

[36] A.-P. Arrigo, W. J. J. Firdaus, G. Mellier et al., "Cytotoxic effects induced by oxidative stress in cultured mammalian cells and protection provided by Hsp27 expression," Methods, vol. 35, no. 2, pp. 126-138, 2005.

[37] A. L. Bryantsev, S. Y. Kurchashova, S. A. Golyshev et al., "Regulation of stress-induced intracellular sorting and chaperone function of Hsp27 (HspB1) in mammalian cells," Biochemical Journal, vol. 407, no. 3, pp. 407-417, 2007.

[38] J. L. Kofron, P. V. Kuzmic, E. Kishore, E. Colon-Bonilla, and D. H. Rich, "Determination of kinetic constants for peptidyl prolyl cis-trans isomerases by an improved spectrophotometric assay," Biochemistry, vol. 30, no. 25, pp. 6127-6134, 1991.

[39] A. Galat, "Peptidylproline cis-trans-isomerases: immunophilins," European Journal of Biochemistry, vol. 216, no. 3, pp. 689707, 1993.

[40] D. A. Fruman, P. E. Mather, S. J. Burakoff, and B. E. Bierer, "Correlation of calcineurin phosphatase activity and programmed cell death in murine T cell hybridomas," European Journal of Immunology, vol. 22, no. 10, pp. 2513-2517, 1992.

[41] J. Liu, J. O. Farmer Jr., W. S. Lane, J. Friedman, I. Weissman, and S. L. Schreiber, "Calcineurin is a common target of cyclophilincyclosporin A and FKBP-FK506 complexes," Cell, vol. 66, no. 4, pp. 807-815, 1991.

[42] L. Gotloib, A. Shostak, V. Wajsbrot, and R. Kushnier, "High glucose induces a hypertrophic, senescent mesothelial cell phenotype after long in vivo exposure," Nephron, vol. 82, no. 2 , pp. 164-173, 1999. 
[43] J. Witowski, A. Jörres, K. Korybalska et al., "Glucose degradation products in peritoneal dialysis fluids: do they harm?" Kidney International, Supplement, vol. 63, no. 84, pp. S148-S151, 2003.

[44] J. D. Williams, K. J. Craig, N. Topley et al., "Morphologic changes in the peritoneal membrane of patients with renal disease," Journal of the American Society of Nephrology, vol. 13, no. 2, pp. 470-479, 2002.

[45] M. N. Schilte, J. W. A. M. Celie, P. M. Ter Wee, R. H. J. Beelen, and J. van den Born, "Factors contributing to peritoneal tissue remodeling in peritoneal dialysis," Peritoneal Dialysis International, vol. 29, no. 6, pp. 605-617, 2009. 


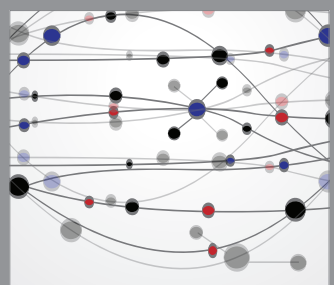

The Scientific World Journal
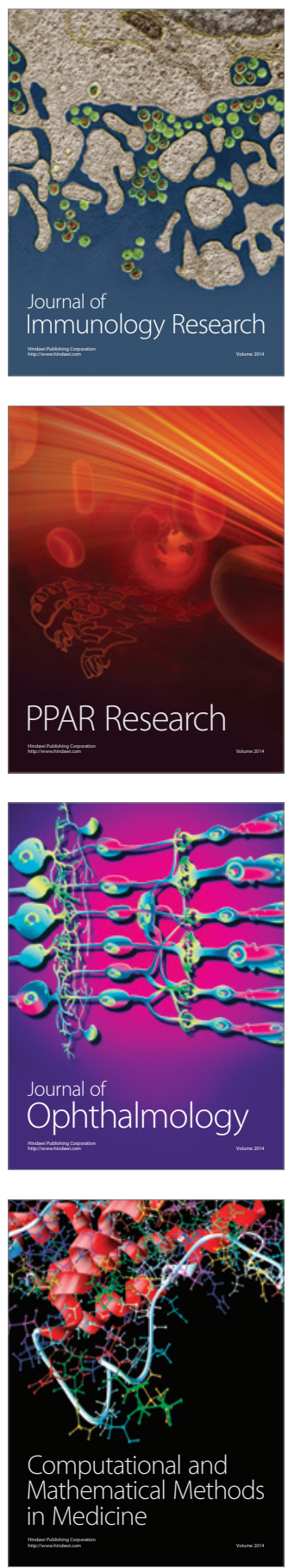

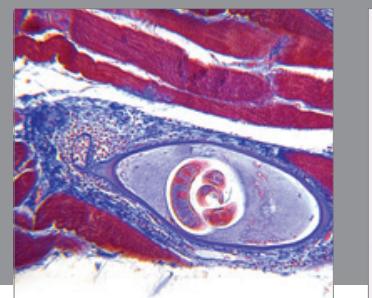

Gastroenterology

Research and Practice
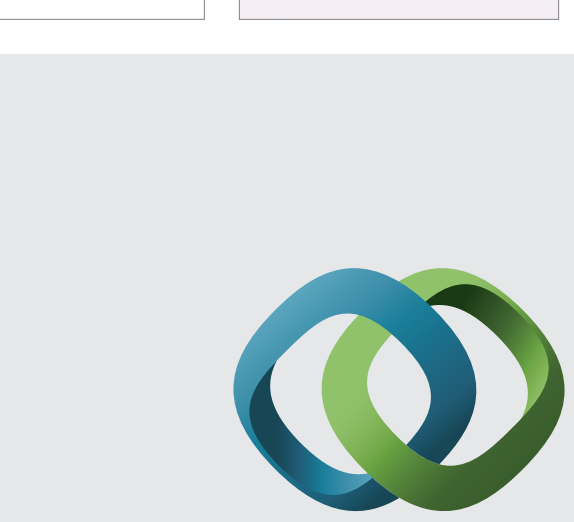

\section{Hindawi}

Submit your manuscripts at

http://www.hindawi.com
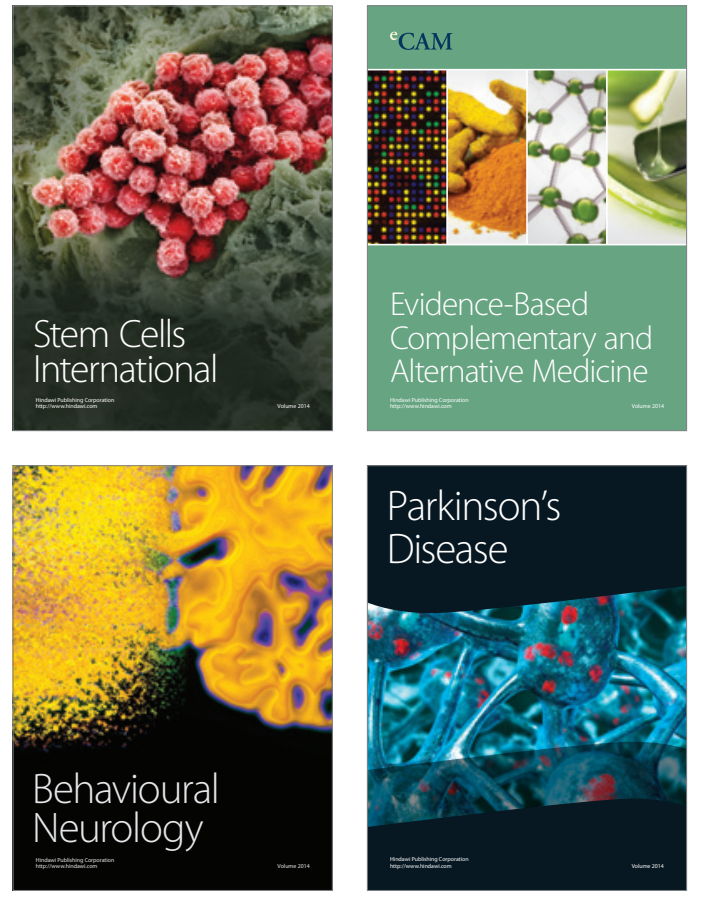
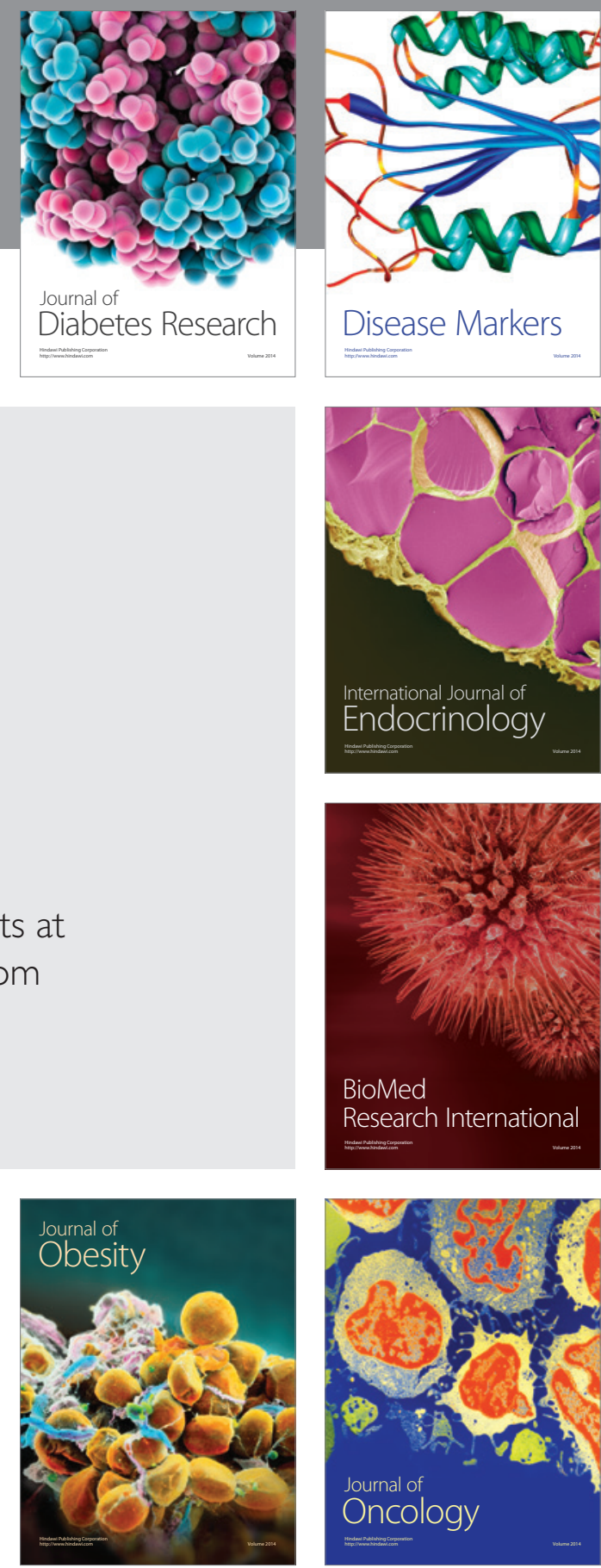

Disease Markers
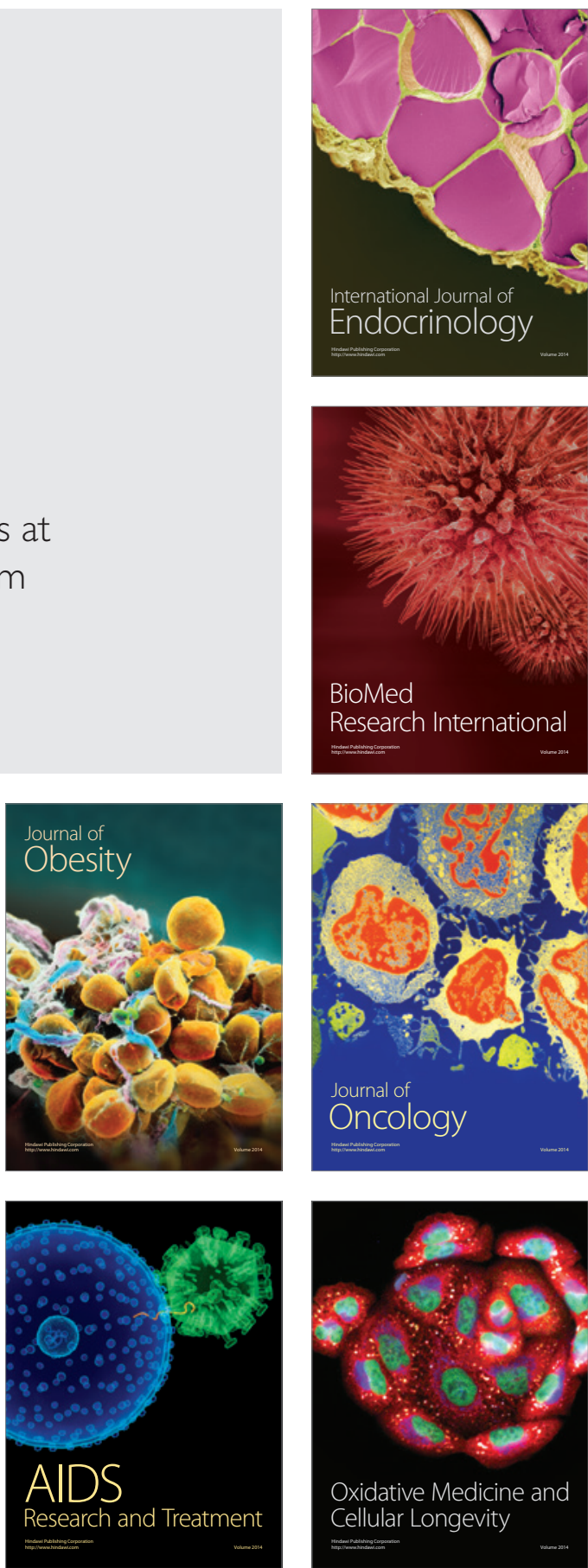